\title{
Genetic and Pharmacological Modulation of the Steroid Sulfatase Axis Improves Response Control; Comparison with Drugs Used in ADHD
}

\author{
William Davies ${ }^{1,2,3,5}$, Trevor Humby ${ }^{1,2,3,5}$, Simon Trent ${ }^{1,2,3}$, Jessica B Eddy ${ }^{1,2}$, Obah A Ojarikre ${ }^{4}$ and \\ Lawrence S Wilkinson*, I,2,3
}

'Behavioral Genetics Group, Schools of Psychology and Medicine, Cardiff University, Cardiff, UK; ${ }^{2}$ MRC Centre for Neuropsychiatric Genetics and Genomics and Institute of Psychological Medicine and Clinical Neurosciences, School of Medicine, Cardiff University, Cardiff, UK; ${ }^{3}$ Neuroscience and Mental Health Research Institute, Cardiff University, Cardiff, UK; ${ }^{4}$ MRC National Institute for Medical Research, London, Cardiff, UK

Maladaptive response control is a feature of many neuropsychiatric conditions, including attention deficit hyperactivity disorder (ADHD). As ADHD is more commonly diagnosed in males than females, a pathogenic role for sex-linked genes has been suggested. Deletion or point mutation of the $X$-linked STS gene, encoding the enzyme steroid sulfatase (STS) influences risk for ADHD. We examined whether deletion of the Sts gene in the $39, X^{Y^{*}} \bigcirc$ mouse model, or pharmacological manipulation of the STS axis, via administration of the enzyme substrate dehydroepiandrosterone sulfate or the enzyme inhibitor COUMATE, influenced behavior in a novel murine analog of the stopsignal reaction time task used to detect inhibitory deficits in individuals with ADHD. Unexpectedly, both the genetic and pharmacological treatments resulted in enhanced response control, manifest as highly specific effects in the ability to cancel a prepotent action. For all three manipulations, the effect size was comparable to that seen with the commonly used ADHD therapeutics methylphenidate and atomoxetine. Hence, converging genetic and pharmacological evidence indicates that the STS axis is involved in inhibitory processes and can be manipulated to give rise to improvements in response control. While the precise neurobiological mechanism(s) underlying the effects remain to be established, there is the potential for exploiting this pathway in the treatment of disorders where failures in behavioral inhibition are prominent.

Neuropsychopharmacology (2014) 39, 2622-2632; doi:I0.I038/npp.20I4.II5; published online I8 June 2014

\section{INTRODUCTION}

Deficits in impulse control are seen in many psychiatric illnesses, ranging from substance abuse, to pathological gambling, to neurodegenerative conditions (Brewer and Potenza, 2008). Maladaptive responding is also a prominent, though nonspecific, feature of attention deficit hyperactivity disorder (ADHD), a neurodevelopmental condition that affects up to $6 \%$ of the population and that can have significant long-term adverse consequences (Brewer and Potenza, 2008; Thapar et al, 2005). Aberrant response inhibition in ADHD has consistently been demonstrated in the stop-signal reaction time task (SSRTT), an assay of the ability to cancel an ongoing motor action in response to a 'stop-signal' (Crosbie et al, 2008).

ADHD is more frequently diagnosed in males than in females (Holden, 2005), suggesting potential contributory

*Correspondence: Professor LS Wilkinson, School of Psychology, Cardiff University, Tower Building, Cardiff CFIO 3AT, UK, Tel: +44 (0)292087 0357, Fax: +44 (0)292087 4858,

E-mail: wilkinson!@cardiff.ac.uk

${ }^{5}$ These authors contributed equally to this work.

Received I October 2013; revised 10 April 20I4; accepted 2 May

2014; accepted article preview online 20 May 2014 sex-linked genetic mechanisms. Cytogenetic deletions encompassing the X-linked steroid sulfatase (STS) gene, or inactivating point mutations within it, result in an elevated rate of ADHD in comparison with the general population (Kent et al, 2008), and there is some evidence that polymorphisms within STS may influence ADHD risk and symptoms (Brookes et al, 2010, 2008; Stergiakouli et al, 2011). The STS enzyme cleaves sulfate groups from a variety of steroids (notably dehydroepiandrosterone sulfate, DHEAS) and the substrates and products of the enzyme, and their multiple downstream effectors, can influence diverse neurobiological processes, via effects on the biosynthesis of androgens and oestrogens and on key neurotransmitter signaling systems (Reed et al, 2005). Furthermore, STS is expressed in regions of the developing and mature human brain known to be important in impulse control, and whose structure/function is frequently perturbed in cases of ADHD, notably frontal cortex, basal ganglia and thalamus (Hart et al, 2013; Perumal and Robins, 1973; Stergiakouli et al, 2011).

Here, we exploited our recent development of a murine analog of the human SSRTT (Humby et al, 2013) to investigate the effects of manipulating the STS axis on response control in mouse models. To assess the effects of gene 
deletion, we used the $39, \mathrm{X}^{\mathrm{Y}^{*}} \mathrm{O}$ mouse, in which the pseudoautosomal Sts and Asmt genes (encoding STS and acetylserotonin $O$-methyltransferase, respectively) are deleted as consequence of an end-to-end fusion of the $\mathrm{X}$ and $\mathrm{Y}$ chromosomes (Trent et al, 2013). We have shown that $39, \mathrm{X}^{\mathrm{Y}^{*}} \mathrm{O}$ mice exhibit diverse behavioral and endocrine phenotypes, including deficits in stimulus detection in the five-choice serial reaction time task consistent with impaired functioning in divided attention (Davies et al, 2009), hyperactivity, heightened emotional reactivity, aggression (Trent et al, 2012b), increased perseveration (Trent et al, 2012a, 2013), and reduced DHEA levels (Trent et al, 2012b, 2013); we have suggested these findings overlap with some of the symptoms seen in ADHD. Here, we augmented the genetic approach with acute pharmacological manipulations involving systemic administrations of the STS substrate DHEAS and the enzyme inhibitor COUMATE (Reed et al, 2005). These additional manipulations were undertaken to confirm that any behavioral effects in the $39, \mathrm{X}^{\mathrm{Y}^{*}} \mathrm{O}$ mouse were due to loss-of-function of STS (rather than loss-of-function of ASMT) and to determine whether the STS-dependent effects were likely to be due to the enzyme's developmental role alone, or due to its ongoing activity into adulthood. Our initial prediction, based on the existing human literature linking STS to ADHD risk, was that STS loss or inhibition would result in impaired response control.

We report that, in contrast to this prediction, both the genetic and pharmacological manipulations of STS function led to markedly enhanced response control in the SSRTT, and that these effects were comparable in magnitude and specificity to those seen with the commonly used ADHD therapeutics methylphenidate and atomoxetine. While the neurobiological mechanism(s) mediating these effects remain to be established there is the potential for exploiting this pathway as an alternative to current therapies used to treat disorders where failures in behavioral inhibition are prominent.

\section{MATERIALS AND METHODS}

\section{Subjects}

Wildtype 40,XY MF1 mice (Harlan, UK, aged 3-4 months at start of testing) were used in initial work validating the SSRTT; this was necessary as our previous work used C57BL/6 strain mice (Humby et al, 2013). This group of mice were also used for the drug studies. For the genetic study, $39, \mathrm{X}^{\mathrm{Y}^{\star}} \mathrm{O}$ and $40, \mathrm{XY}$ mice on a predominantly MF1 background were bred, genotyped, and treated prior to behavioral analysis as described previously (Trent et al, 2012a); subjects were aged 8-10 months at start of testing in the SSRTT. $39, \mathrm{X}^{\mathrm{Y}^{*}} \mathrm{O}$ mice were generated from two karyotypically-distinct mothers $\left(39, \mathrm{X}^{\text {Paf }} \mathrm{O}\right.$ or $\left.40, \operatorname{In}(\mathrm{X})^{\mathrm{Paf}} / \mathrm{X}\right)$, and several of the $39, \mathrm{X}^{\mathrm{Y}^{\star}} \mathrm{O}$ mice had to be housed individually owing to their propensity to fight. Therefore, we explicitly tested post hoc for any effects of birth mother, or of single housing, on $39, \mathrm{X}^{\mathrm{Y}^{*}} \mathrm{O}$ behavior. In addition, given that mice for the task specification/drug studies and the genetic studies differed in age, we also considered whether there might be potential confounds in the data owing to ageing effects. All mice were housed in the same vivarium maintained at $21 \pm 2{ }^{\circ} \mathrm{C}$ and $50 \pm 10 \%$ humidity, with a $12 \mathrm{~h}$ light-dark cycle (lights on at 07:00 h), with food and water available ad libitum, unless stated otherwise. Animals were treated in accordance with the Animal (Scientific Procedures) Act (United Kingdom, 1986).

\section{SSRTT: Habituation and Training}

Reinforcer habituation, training, and assessment of SSRTT task performance were as described previously (Humby et al, 2013 and Supplementary Materials and Methods). Briefly, the animals were habituated to liquid reinforcer ( $10 \%$ condensed milk, Nestle Ltd) before undergoing SSRTT training in mouse nine-hole boxes (Campden Cognition, UK). Training to baseline involved shaping the mice to respond sequentially at two stimulus locations, using nosepokes, to give rise to a 'go' response, and then learning to withhold responding to the second stimulus location when an auditory stop-signal was presented, to give rise to a 'stop' response (Figure 1a).

\section{SSRTT: Behavioral Manipulations}

Mice could perform a maximum of 100 trials $(\leq 20 \mathrm{~min}$ session duration), where $80 \%$ of trials were 'go' trials and $20 \%$ were interpolated 'stop' trials. At baseline, on 'stop' trials, the stop-signal was always presented coincident with the beginning of the response (ie, $0 \%$ of the individual correct go reaction time for each subject), making stopping relatively easy, but in separate probe sessions the position of the stop-signal was varied relative to the individual correct go reaction times for each mouse making stopping more or less difficult ie, at $10,20,30,40,50,60,70,80$, and $90 \%$ into the individualized go reaction time for each mouse (Figure 1a). At 90\%, the stop signal was played close to the execution of the response, and hence stopping was most difficult (Humby et al, 2013; Carter et al, 2003). There were at least 3 days at baseline criteria between each probe session, and the order of presentation of these sessions was randomised between subjects.

\section{SSRTT: Pharmacological Manipulations}

As previously described (Humby et al, 2013), drug effects in MF1 mice were determined in sessions with a $50 \%$ stop-signal position, when subjects showed $\sim 50 \%$ correct stopping. At this point, behavior conforms to the prevailing 'race' model of response inhibition, whereby the ability to cancel an ongoing motor action is dependent on competition between dissociable, parallel brain processes of going and stopping (Eagle and Robbins, 2003; Logan, 1994). Methylphenidate $\mathrm{HCl}(n=12$, at doses of $0,0.3,1,1.5$, and $3 \mathrm{mg} / \mathrm{kg}$, Sigma, UK) and atomoxetine $\mathrm{HCl}(n=12$, at doses of $0,0.6,1,3$ and $5 \mathrm{mg} / \mathrm{kg}$, Sigma, UK), at doses shown previously to be effective at modifying rodent SSRTT behavior (Eagle et al, 2007; Humby et al, 2013; Robinson et al, 2008a), were made up in physiological saline and administered $30 \mathrm{~min}$ prior to testing (i.p.). COUMATE was administered at $10 \mathrm{mg} / \mathrm{kg}$ p.o. $24 \mathrm{~h}$ before testing in $0.5 \%$ methylcellulose physiological saline vehicle $(n=11)$, and DHEAS (Sigma, UK) was administered at $40 \mathrm{mg} / \mathrm{kg}$ i.p. in distilled water vehicle $1 \mathrm{~h}$ before testing, i.p. $(n=12)$. 
a
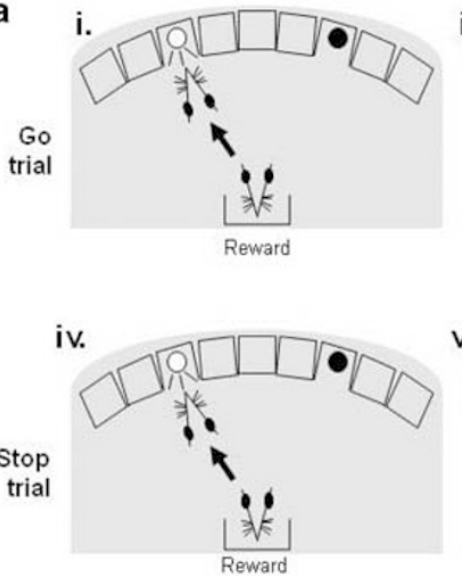

b

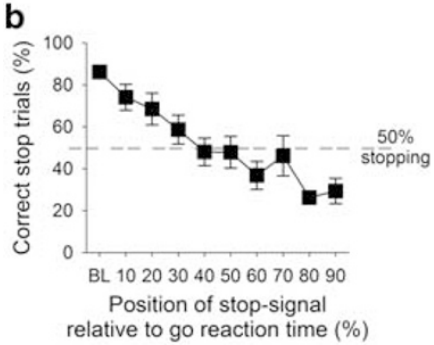

e

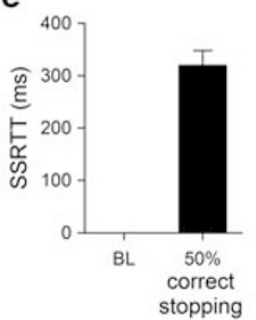

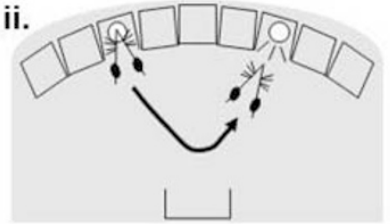

ii.

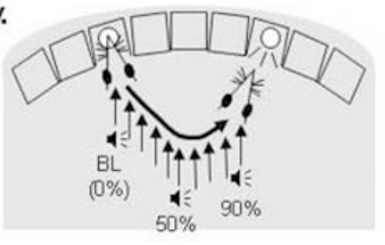

C

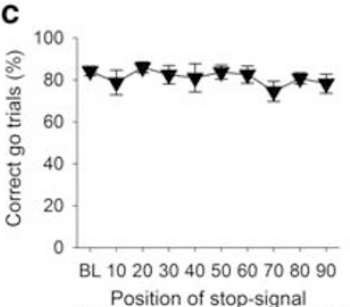

elative to go reaction time $(\%)$

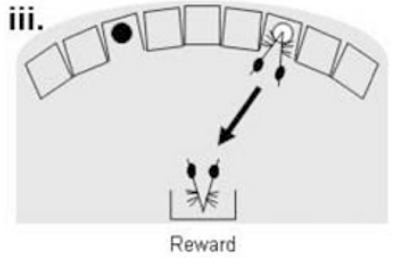

vi.

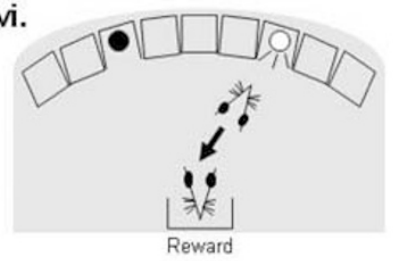

d

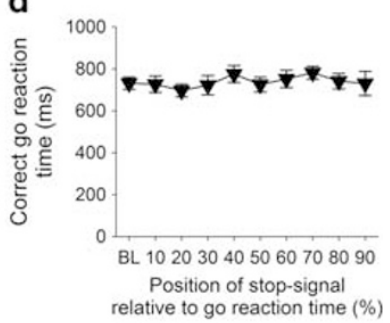

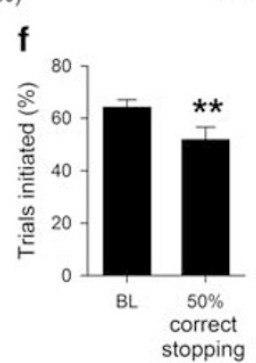
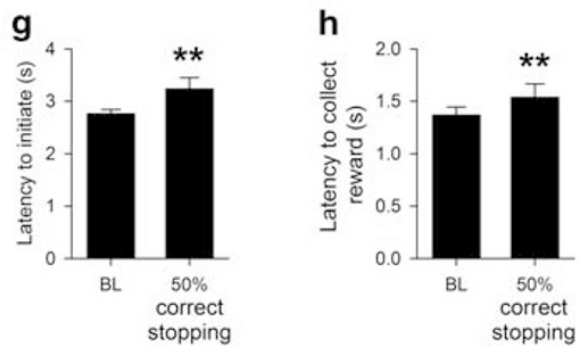

Figure I The stop-signal reaction time task (SSRTT), baseline, and task manipulations in adult MFI 40,XY mice $(n=14)$. The SSRTT consisted of two interpolated trial types: Go trials (80\%), and Stop trials (20\%). Subjects initiated a go trial by a nosepoke response to a stimulus in the left-hand hole (ai); this resulted in the go stimulus (light in right-hand hole) being illuminated (aii). A correct nosepoke response at the go stimulus resulted in the delivery of reinforce (aiii). Stop trials were also initiated by a left nosepoke (aiv), which subsequently resulted in presentation of a light in the right-hand hole (av). However, on these trials an auditory stop signal was presented after trial initiation, instructing the mouse to inhibit responding to the second stimulus (av). Successful stopping resulted in reinforce presentation on these trials (avi). The stop signal was presented at increasing intervals into a mouse's individualized reaction time, with early stop signals (0\%) allowing for easier stopping, and later signals, close to the execution of the second stimulus response (90\%), making stopping more difficult. Assessment of SSRTT performance in MFI mice showed that presenting the auditory 'stop-signal' progressively closer to the end of the individual go response made stopping more difficult (b), but did not affect the proportion of correct go trials (c), or the speed of the go response (d). Further analysis of SSRTT performance was conducted to examine the point where competition between the go and stop responding was at its greatest; thus, data from each subject were ranked and sessions where correct stopping was at $50 \pm 10 \%$ averaged. At this point the SSRT, the latency to withhold responding, was calculated (e). When stopping with $50 \%$ accuracy, there were small but significant changes from baseline (BL) performance in other performance measures, such as a decrease in the proportion of trials initiated $(f)$ and increases in the latency to initiate a trial (g) and the time taken to enter the food magazine following a successful trial (h). *** denotes $P<0.0$ I for significant difference between test condition.

The doses of COUMATE and DHEAS used had previously been shown to specifically affect aspects of cognition in similar operant tasks in mice (Davies et al, 2009). Drugs were given in a Latin square design, where animals were given a single session with each dose of drug and vehicle, with at least four days of stable performance at baseline criteria between each drug treatment to allow for washout.

\section{SSRTT: Measures and Statistical Analyses}

Key measures from the SSRTT were \% correct stop trials, stop-signal reaction time (SSRT), \% correct go trials and correct go reaction time. Ancillary measures of general task performance included overall number of trials initiated, latency to initiate a trial and latency to enter the food magazine following a successful go or stop trial. For a full listing of measures and definitions see Supplementary Materials and Methods. SSRTs in the task were estimated employing the standard procedure described in Logan et al, (1984), using data from where the proportion of correct stop responses is $\sim 50 \%$. For each subject, data from the sessions in which the stop-signal positions were varied relative to the individualized go reaction time, were ranked by the proportion of correct stop responses, and data from 
the sessions in which this value was between $40 \%$ and $60 \%$ (ie, $50 \% \pm 10 \%$ ) were averaged. The latency of stopping as defined by the SSRT was derived from the distribution of correct go reaction times and the proportion of correctly stopped trials as previously described (Eagle and Robbins, 2003; Logan, 1994). Hence, for each of the sessions determined above, the correct go reaction times were rank ordered from smallest to largest and the nth value found, where $n$ is the rank order position based on the proportion of failing to stop correctly in stop trials, corrected for the occurrence of omitted go trials (Eagle and Robbins, 2003; Solanto et al, 2001; Tannock et al, 1989). To determine the SSRT, the time the stop-signal was presented (ie, 'mean correct go reaction time' $\times$ ' $\%$ mean stop-signal position') was subtracted from the nth correct go reaction time value (Supplementary Materials and Methods).

Data were analyzed using SPSS (V.18, SPSS Inc, USA), and were subject to $t$-test or ANOVA. Between-group factors included GENOTYPE $\left(40, \mathrm{XY}\right.$ vs $\left.39, \mathrm{X}^{\mathrm{Y}^{*}} \mathrm{O}\right)$ and DOSE (vehicle and dose of each drug), and within-group factors included STOP-SIGNAL POSITION (0\% (baseline)-90\%) and STOP-PERFORMANCE (baseline responding vs 50\% correct stopping). Scores calculated as percentages were arcsine transformed prior to analysis. Where sphericity assumptions were violated, Greenhouse-Geisser corrections were used. If ANOVA indicated a significant interaction between factors, post hoc pairwise comparisons were performed using Tukey's Honestly Significant Difference test. Criterion level of significance was set at the 0.05 level, and all data are shown as mean \pm SEM.

\section{RESULTS}

\section{Behavioral Specification of the SSRTT in Adult Male MF1 Mice}

The MF1 mice were able to learn and perform the task to the same high degree of performance seen previously in C57BL/ 6 strain mice (Humby et al, 2013). On average, it took $\sim 34$ sessions to achieve stable baseline performance (defined as $>70 \%$ initiated trials, $>80 \%$ correct go responses and $>80 \%$ correct stopping performance). At baseline, where the presentation of the auditory stop-signal occurred at start of the go response, mice demonstrated high levels of stimulus control with rapid $(2.7 \pm 0.1 \mathrm{~s})$ and high $(74.4 \pm 3.1 \%)$ levels of responding to the initiating stimulus, go latencies comparable to those reported for rat and human subjects $(731 \pm 29 \mathrm{~ms})$ (DeVito et al, 2009; Eagle and Robbins, 2003), and high levels of successful stopping during stop trials $(86.4 \pm 3.1 \%)$ and general motivation, as indexed by the rapid collection of reward following a successful trial (1.4 $\pm 0.1 \mathrm{~s})$ (Supplementary Table S1).

The effects of altering the position of the auditory stopsignal during stop trials are illustrated in Figure $1 \mathrm{~b}-\mathrm{h}$. Making stopping more difficult had the predicted effect on the ability of the mice to withhold responding, decreasing the proportion of correctly stopped trials (Figure $1 \mathrm{~b}$, effect of STOP-SIGNAL POSITION, $\left.\mathrm{F}_{9,117}=11.14, P=0.0001\right)$, but importantly not affecting correct go responding (Figure 1c, effect of STOP-SIGNAL POSITION, $\mathrm{F}_{9,117}=1.56, P=0.14$ ) or go latencies in the interpolated go trials (Figure 1d, effect of STOP-SIGNAL POSITION, $\left.\mathrm{F}_{9,117}=1.48, P=0.17\right)$. The mean position of the stop-signal that resulted in $50 \%$ correct stopping was $48.19 \pm 5.6 \%$ into the mean go responses times for these mice; at this point, mean SSRT was $319 \pm 28 \mathrm{~ms}$ (Figure 1e), similar to that reported in human and rat tasks and C57BL/6 mice (Eagle et al, 2007; Humby et al, 2013; Tannock et al, 1989). There were the modest, but significant, STOP-PERFORMANCE effects in some of the ancillary task measures, such as decreased proportion of trials initiated at $50 \%$ correct responding (Figure 1f, $t_{13}=$ 3.92, $P=0.002)$ together with increased latencies to initiate a trial (Figure 1g, $t_{13}=-2.48, P=0.027$ ) and collect the reinforcer (Figure $1 \mathrm{~h}, t_{13}=-2.45, P=0.029$ ).

\section{Effects of the Clinically Effective Drugs Methylphenidate and Atomoxetine on SSRTT Performance in Adult Male MF1 Mice}

Consistent with our previous work in C57BL/6 mice (Humby et al, 2013), and that of others in rats (Eagle et al, 2007; Robinson et al, 2008a), the clinically effective drugs methylphenidate and atomoxetine enhanced stopping ability (Figure 2). There was a significant effect of methylphenidate administration on successful stopping (Figure 2a, effect of DOSE, $\mathrm{F}_{4,44}=2.67, P=0.04$ ) and SSRT (Figure $2 \mathrm{~b}$, effect of DOSE, $\mathrm{F}_{4,44}=11.09, P=0.001$ ), consistent with quicker and more effective stopping. Drug effects on stopping were highly specific with no effects on correct go responding (Figure $2 c$, effect of DOSE $\mathrm{F}_{4,44}=0.97, P=0.43$ ) or go latency (Figure 2d, effect of DOSE, $\mathrm{F}_{4,44}=1.06$, $P=0.39$ ). Moreover, there were no significant effects of methylphenidate on other general features of SSRTT behavior (Supplementary Figure S2). Administration of atomoxetine also increased successful stopping (Figure 2e, effect of DOSE, $\left.\mathrm{F}_{4,44}=7.46, P=0.001\right)$ and decreased SSRT, relative to vehicle treatment (Figure 2f, effect of DOSE, $\left.\mathrm{F}_{4,44}=4.11, P=0.01\right)$. Atomoxetine administration did not affect the go response (Figure 2g-h, effect of DOSE, $\mathrm{F}_{4,44}=1.67, P=0.18$ and $\mathrm{F}_{4,44}=0.90, P=0.47$, for correct go responding and go latency, respectively); atomoxetine administration at all doses tended to reduce the proportion of trials initiated, there were no significant effects on ancillary measures (Supplementary Figure S2).

\section{Enhanced Response Inhibition in $39, \mathrm{X}^{\mathrm{Y}^{*}} \mathrm{O}$ Mice Relative to $40, \mathrm{XY}$ Mice}

$39, \mathrm{X}^{\mathrm{Y}^{*}} \mathrm{O}$ mice were equivalent to $40, \mathrm{XY}$ controls in terms of reinforcer preference, and progression through the training stages of the SSRTT, reaching stable baseline performance in $\sim 39$ sessions (Supplementary Table S2). Both groups of mice showed high levels of stimulus control at baseline, initiating $>70 \%$ of trials, achieving $>80 \%$ go and stop responses with rapid go reaction times $(\sim 700 \mathrm{~ms})$; both groups also showed efficient patterns of behavior in relation to nose-poke responses/trial initiation (40,XY: $1.12 \pm 0.02 v s$ $\left.39, \mathrm{X}^{\mathrm{Y}^{*}} \mathrm{O}: 1.15 \pm 0.02\right)$ and the rapid collection of reward following a successful trial $\left(40, \mathrm{XY}: 1.98 \pm 0.36 \mathrm{~s} v s 39, \mathrm{X}^{\mathrm{Y}} \mathrm{O}\right.$ : $1.88 \pm 0.33 \mathrm{~s}$ ) (Supplementary Table S2).

Altering the position of the auditory stop-signal during stop trials, by presenting the auditory stop stimulus progressively closer to the execution of the go response, led to systematic reductions in the ability to stop for both 

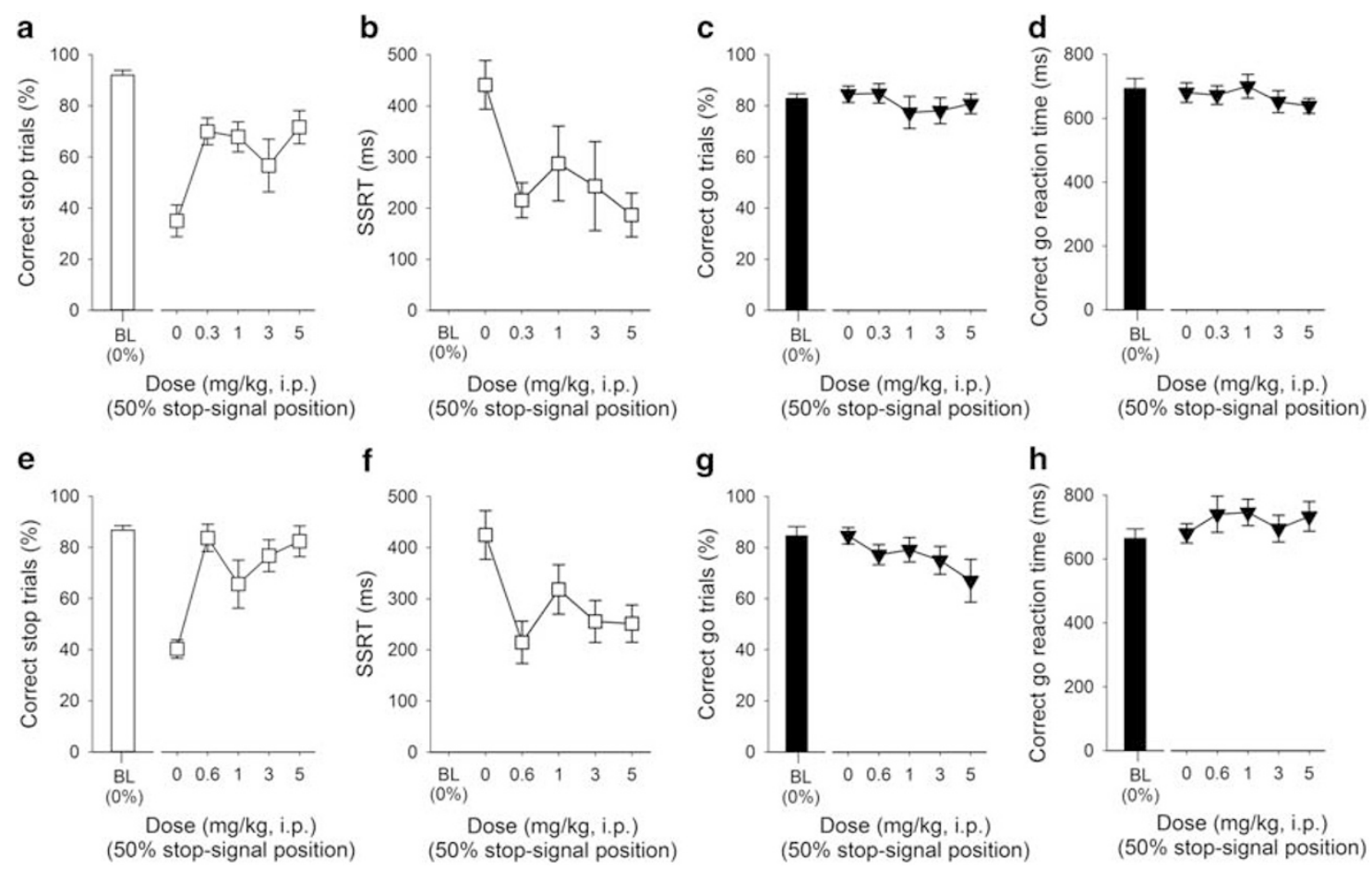

Figure 2 Effects of methylphenidate and atomoxetine administration on stop-signal reaction time task performance in adult MFI 40,XY mice $(n=12)$. At all doses used, methylphenidate improved stopping performance (a) and reduced the SSRT (b), but did not affect the proportion of correct go trials (c) or the latency to make a correct go response (d). Administration of atomoxetine increased successful stopping (e) and decreased the SSRTT (f). The specificity of atomoxetine to affect stopping was shown by a lack of effects on the proportion of correct go trials (g) and speed of the go response ( $h$ ). Baseline data (BL ie, mean of the five sessions immediately preceding each drug treatment session) when the stopsignal presentation were concurrent with the start of the go response (0\%) are shown for illustrative purposes and were not included in the statistical analysis.

groups of mice (Figure 3a, effect of STOP-SIGNAL POSITION, $\left.\mathrm{F}_{9,225}=25.46, P=0.001\right)$. However, $39, \mathrm{X}^{\mathrm{Y}^{*}} \mathrm{O}$ mice were less affected by this task manipulation (interaction of GENOTYPE $\mathrm{x}$ STOP-SIGNAL POSITION, $\mathrm{F}_{9,225}=$ 3.32, $P=0.001$ ), confirmed by pairwise comparisons showing that successful stopping was significantly different between $40, \mathrm{XY}$ and $39, \mathrm{X}^{\mathrm{Y}^{*}} \mathrm{O}$ mice $(P<0.05)$ when the stopsignal was presented at $30-70 \%$ positions, but not at baseline ( $0 \%), 10 \%, 20 \%, 80 \%$ or $90 \%$ stop-signal positions. In contrast, there were no significant between-group differences in correct go responses (Figure $3 \mathrm{~b}$, effect of GENOTYPE, $\left.\mathrm{F}_{1,25}=1.48, P=0.24\right)$ or in correct go reaction times (Figure 3c, effect of GENOTYPE, $\mathrm{F}_{1,25}=2$. 39, $P=0.13$ ).

Calculation of the SSRT at the 50\% correct stopping point showed that $39, \mathrm{X}^{\mathrm{Y}^{*}} \mathrm{O}$ mice had significantly quicker stop latencies than $40, \mathrm{XY}$ mice (Figure $3 \mathrm{~d}, t_{25}=3.24, P=0.01$ ). Together with the increased proportion of correctly stopped trials, these data indicated more rapid and effective stopping in the $39, \mathrm{X}^{\mathrm{Y}^{*}} \mathrm{O}$ mice. $39, \mathrm{X}^{\mathrm{Y}^{*}} \mathrm{O}$ mice initiated more trials than $40, \mathrm{XY}$ mice at baseline and when they were at the $50 \%$ correct stopping point (Figure 3e, effect of GENOTYPE, $\mathrm{F}_{1,25}=12.62, P=0.002$ ), but were not quicker to start a trial (Figure 3f, effect of GENOTYPE, $\mathrm{F}_{1,25}=3.98, P=0.06$ ), or to collect the reinforcer (Figure $3 \mathrm{~g}$, effect of GENOTYPE, $\left.\mathrm{F}_{1,25}=2.31, P=0.14\right)$. There were no differences in these measures at baseline or $50 \%$ correct stopping performance (effect of STOP-SIGNAL POSITION, $\mathrm{F}_{1,25}=0.56, P=0.46$,
$\mathrm{F}_{1,25}=2.29, P=0.14, \mathrm{~F}_{1,25}=0.93, P=0.35$ for the proportion of initiated trials, latency to initiate a trial and latency to collect the reinforcer, respectively). The clear dissociations between $39, \mathrm{X}^{\mathrm{Y}^{*}} \mathrm{O}$ effects on stopping and going behaviors made it unlikely that the enhanced stopping in $39, \mathrm{X}^{\mathrm{Y}^{*} \mathrm{O}}$ mice was due to adoption of some qualitatively distinct behavioral strategy (eg, postural), but to be sure, the lack of any such difference was confirmed by video analysis. $39, \mathrm{X}^{\mathrm{Y}^{*}} \mathrm{O}$ mice were generated from two karyotypicallydistinct mothers, and some of these mice had to be individually housed during testing. The data were indifferent to either of these potentially confounding factors, insofar as the key SSRTT measures did not differ between $39, \mathrm{X}^{\mathrm{Y}^{*}} \mathrm{O}$ mice from the two types of mother (Supplementary Table S3), nor between singly and group-housed $39, \mathrm{X}^{\mathrm{Y}^{\star}} \mathrm{O}$ mice (Supplementary Table S4).

\section{Pharmacological Manipulation of STS Function also Enhances Response Inhibition in Adult Male MF1 Mice}

Treatment with both the enzyme inhibitor COUMATE (Figure 4) and its substrate DHEAS (Figure 5) enhanced inhibition, as evidenced by significant DOSE effects on successful stopping (Figure $4 \mathrm{a}, t_{10}=-4.53, P=0.001$; Figure $5 \mathrm{a}, t_{11}=-5.59, P=0.0001$, respectively) and more rapid SSRTs (Figure $4 \mathrm{~b}, t_{10}=3.10, P=0.011$; Figure $5 \mathrm{~b}$, $t_{11}=2.44, P=0.03$, respectively). In the case of COUMATE, the effects on stopping were not accompanied by changes in 

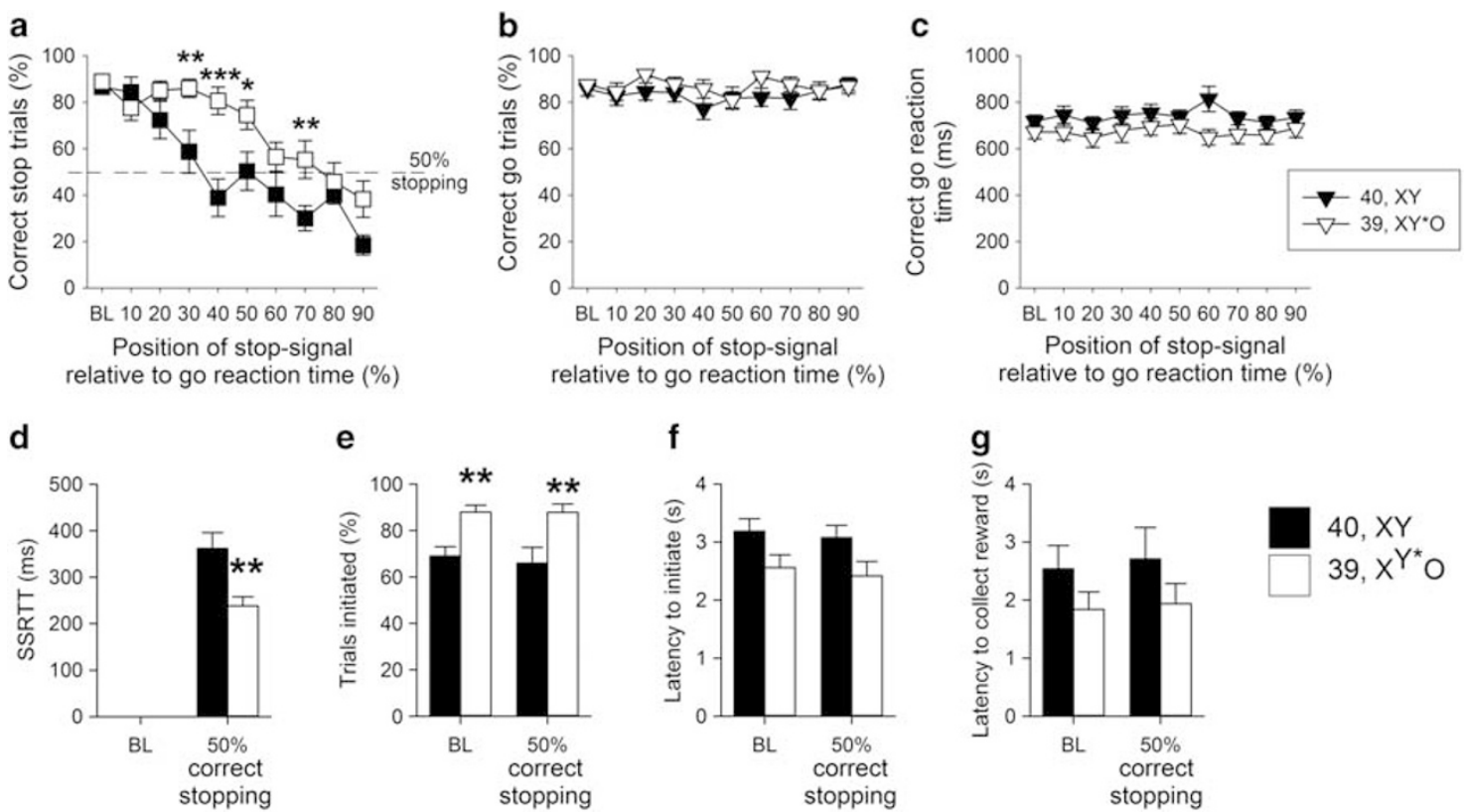

Figure 3 Comparison of stop-signal reaction time task performance in $39, X^{Y *} O(n=16)$ and $40, X Y(n=I I)$ adult MFI male mice. Altering the stopsignal position led to a systematic decrease in the ability of all the mice to stop, although $39, X^{Y *} \bigcirc$ mice were less affected by this manipulation than $40, X Y$ mice (a). The effects of the genetic manipulation were highly specific, in that there were no between-group differences in the proportion of correct trials (b) or speed of correct responding (c). $39, X^{Y *} O$ mice were quicker to stop than $40, X Y$ control mice, reflected by a decrease in their SSRT (d). 39, $X^{Y^{*}} O$ mice initiated more trials than 40,XY mice, both at baseline and at 50\% correct stopping (e). There were no significant group differences in the latencies to initiate a trial $(f)$ or to collect the reinforcer $(g)$. ****, *** and * denote $P<0.001, P<0.01$ and $P<0.05$, respectively for significant pairwise differences between groups.

going behaviors (Figure $4 \mathrm{c}, t_{10}=-1.89, P=0.20$ for correct go responding and Figure $4 \mathrm{~d}, t_{10}=-0.24 . P=0.81$, for the go latency) or on other general features of behavior, such as the overall trials initiated (Figure $4 \mathrm{e}, t_{10}=-0.22, P=0.83$ ), trial initiation latency (Figure $4 \mathrm{f}, t_{10}=1.01, P=0.33$ ), or the reinforcer collection latency (Figure $4 \mathrm{~g}, t_{10}=-2.21$, $P=0.06)$. As with COUMATE, there were no effects of DHEAS on correct go responding (Figure $5 c, t_{11}=0.22$, $P=0.83$ ), the latency in go trials (Figure $5 \mathrm{~d}, t_{11}=-1.13$. $P=0.28$ ), or trial initiation latency (Figure $5 \mathrm{f}, t_{11}=2.09$, $P=0.06)$; however, there was a significant reduction in the number of trials initiated (Figure $5 \mathrm{e}, t_{11}=5.41, P=0.0001$ ) and an increase in the reinforcer collection latency (Figure 5g, $t_{11}=-3.23, P=0.008$ ).

\section{DISCUSSION}

We report novel effects of manipulating the STS axis on response control assayed in a recently developed murine analog of the SSRTT, which permits a high degree of translation between human clinical populations and rodent models (Humby et al, 2013; Humby and Wilkinson, 2011). The MF1 outbred mice used in the present studies were able to learn and perform the SSRTT, exhibiting high levels of stimulus control. Furthermore, as in people, performance was dissociable in terms of going and stopping, and was, therefore, consistent with the prevailing 'race' model of behavioral inhibition whereby action cancelation is dependent on competition between discrete brain processes of going and stopping (Logan, 1994). Additionally, stopping abilities were enhanced following systemic administration of the mixed dopamine/noradrenaline reuptake inhibitor methylphenidate and the more specific noradrenaline reuptake inhibitor atomoxetine, recapitulating the effectiveness of these drugs on response control in clinical and healthy human populations (Aron et al, 2003; Chamberlain et al, 2006; DeVito et al, 2009; Tannock et al, 1989).

A main finding of the current work was the inhibitionpromoting effects of the genetic mutation in the $39, \mathrm{X}^{\mathrm{Y}^{*}} \mathrm{O}$ mice. This was not predicted on the basis of the existing ADHD/STS clinical literature but was consistent, in part, with previous data showing reduced premature responding in these same mutants in the five-choice serial reaction time task (Davies et al, 2009). Importantly, the present findings were highly specific, and in particular unlikely to be confounded by the increased activity and 'response rate' phenotypes previously reported in $39, \mathrm{X}^{\mathrm{Y}^{*}} \mathrm{O}$ mice (Trent et al, 2012b, 2013) in that these phenotypes might be anticipated to be associated with greater difficulty in stopping. Moreover, go response latencies, which were likely to have been affected by between-group differences in nonspecific factors such as activity, were equivalent across $40, \mathrm{XY}$ and $39, \mathrm{X}^{\mathrm{Y}^{\star}} \mathrm{O}$ genotypes.

We sought confirmation of the genetic finding by testing the effects of acute enzyme inhibition using COUMATE at a dose previously shown to inhibit brain activity in mice by $\sim 70 \%$ but to have no effect on locomotor activity (Davies et al, 2009; Nicolas et al, 2001). This experiment was an important control to confirm that the phenotype in the $39, \mathrm{X}^{\mathrm{Y}^{*}} \mathrm{O}$ mice was due solely to STS deficiency (rather than lack of acetylserotonin $\mathrm{O}$-methyltransferase; the other gene 
a

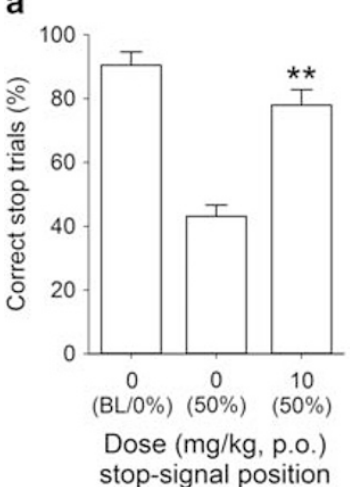

e

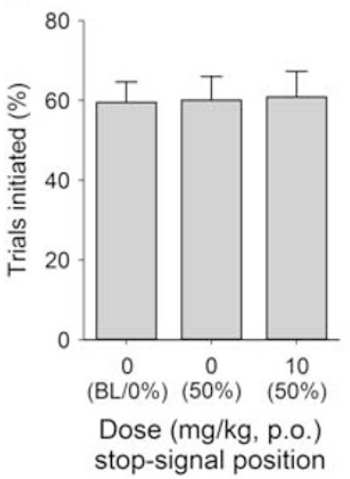

b

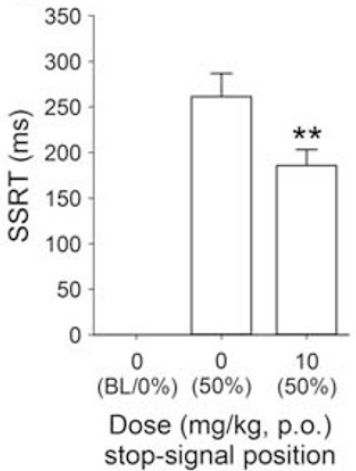

f

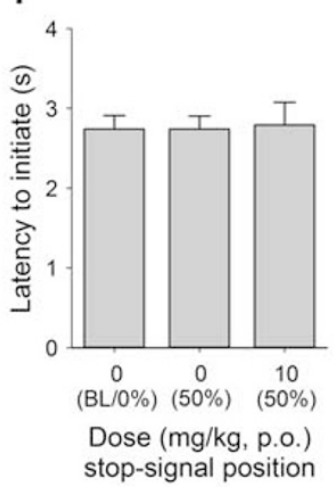

c

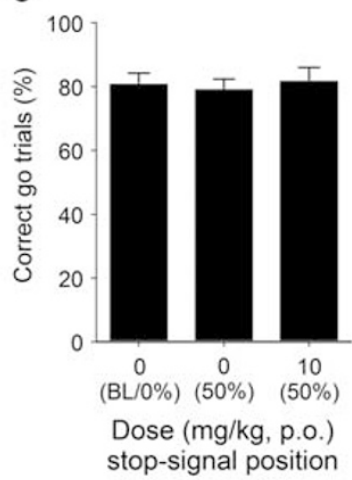

g

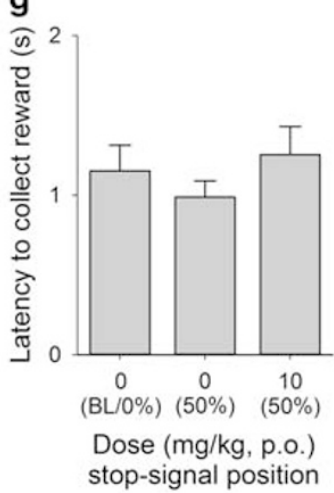

d

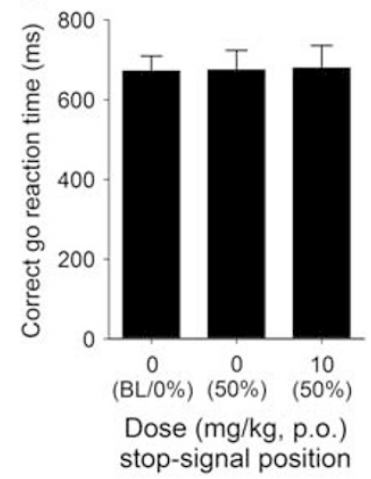

Figure 4 Effects of COUMATE administration $(10 \mathrm{mg} / \mathrm{kg})$ on stop-signal reaction time task performance in adult male MFI mice $(n=\mid \mathrm{I})$. COUMATE treatment increased the proportion of correctly stopped trials (a) and decreased the SSRT (b). Effects of COUMATE were specific to the stop response, as there were no effects on the proportion of correct go trials performed (c) or correct go reaction time (d); nor were there any group effects on the number (e) or latency ( $f$ ) to initiate trials, or in the latency to collect reinforcer (g). Baseline data (BL ie, mean of the five sessions immediately preceding each drug treatment session) when the stop-signal presentation were concurrent with the start of the go response (0\%) are shown for illustrative purposes and were not included in the statistical analysis. ${ }^{*} * *$ and $* *$ denote $P<0.01$ and $P<0.00 \mathrm{I}$, respectively, for significant pairwise differences between drug treatments.

deleted in $39, \mathrm{X}^{\mathrm{Y}^{*}} \mathrm{O}$ mice (Trent et al, 2013) and also allowed us to distinguish whether the behavioral effects arose owing to the developmental and/or ongoing effects of enzyme deficiency. We found that acute administration of COUMATE had the same highly specific effects as the constitutive genetic lesion in the $39, \mathrm{X}^{\mathrm{Y}^{*}} \mathrm{O}$ mice (ie, enhancing stopping abilities in the absence of effects on going behaviors) consistent with an effect of acute regulation of STS on response inhibition in adult brain. The converging evidence from genetic and pharmacological approaches argued strongly against the possibility that the $39, \mathrm{X}^{\mathrm{Y}^{*}} \mathrm{O}$ data were confounded in some way by the mother's karyotype or by the requirements for single housing in some cases. Similarly, the convergent data across the group of younger adult mice used for the task specification/pharmacological studies and older adult mice used for the genetic studies, together with the general similarity in behavioral performance across the groups, indicated the data were not confounded by age effects.

We further probed the effects of acute manipulation of the STS axis by administration of the enzyme substrate DHEAS at a dose reported to have no effects on locomotor activity (Davies et al, 2009). Again, this treatment led to enhanced stopping performance without influencing going trials or most general aspects of behavior (although the drug did reduce overall number of trials initiated). Administration of DHEAS at the same dose and route used in the present work, results in elevated brain levels of both DHEAS and DHEA (the direct product of STS action on DHEAS) within one hour in male mice (Nicolas and Fry, 2007). Therefore, the DHEAS effects on stopping that we observed could have been the result of increased levels of DHEAS and/or DHEA in the brain. However, given that the effects of DHEAS administration on stopping resemble those elicited by enzyme inhibition and STS gene deletion, and the latter two manipulations impair the conversion of DHEAS to DHEA (Nicolas and Fry, 2007; Rhodes et al, 1997; Trent et al, 2013), the common causal factor in enhancing control of responding in the SSRTT across the three experimental procedures was most likely to have been increased DHEAS levels.

Our current and previous data using animal models reveal a consistent pattern of effects, whereby impaired, or absent, STS function leads to both enhanced response control and attentional deficits (Davies et al, 2007, 2009). In rodents, attentional and impulsivity phenotypes are dissociable both psychologically and in terms of discrete underlying neural substrates (Bari and Robbins, 2013; Robbins, 2002), and work by Blondeau and Dellu-Hagedorn (2007) has shown individual differences whereby the same 
a

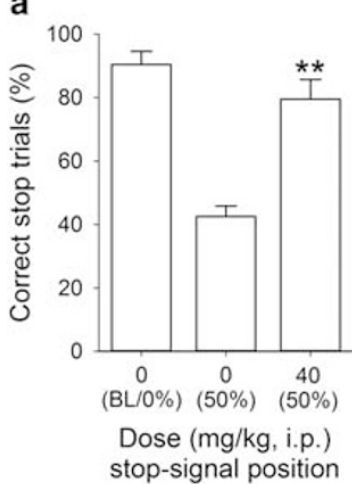

e

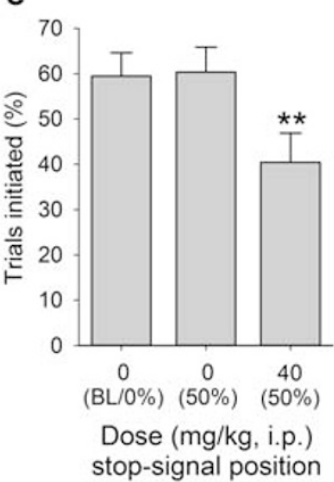

b

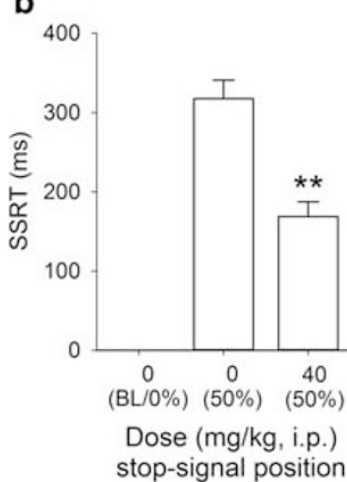

f

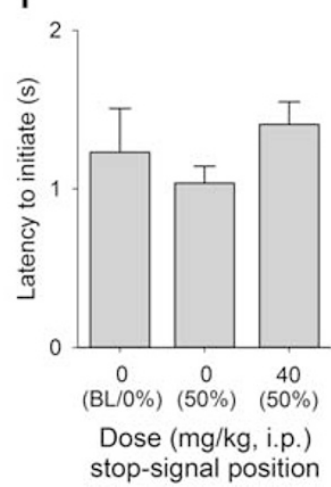

C
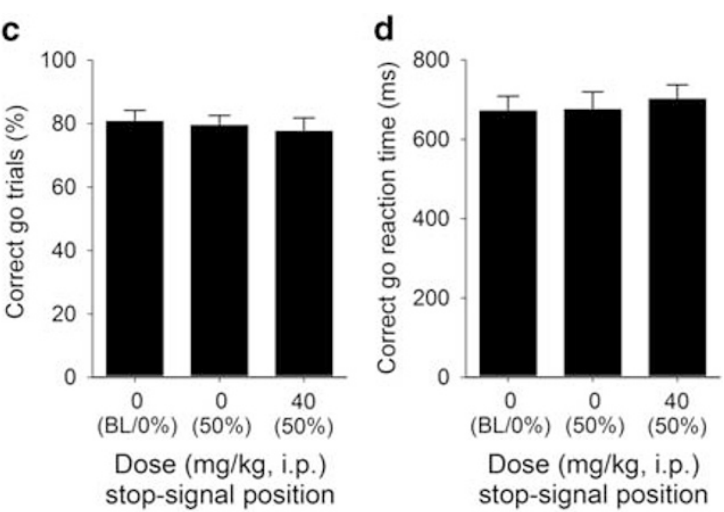

g

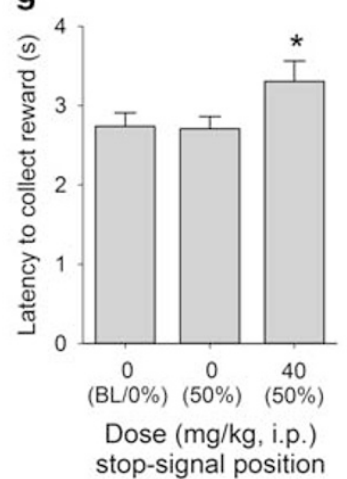

Figure 5 Effect of dehydroepiandrosterone sulfate (DHEAS) administration (40 mg/kg) on stop-signal reaction time task performance in adult male MFI mice $(n=12)$. Administration of DHEAS increased the proportion of correctly stopped trials (a) and decreased SSRT (b). Although there was no effect of drug treatment on the correct go response measure (c) or on the speed of the correct go response (d), DHEAS administration did result in effects on general task performance, such as reducing the number of trials initiated (e) and increasing reinforcer collection latency (g); there was also a near-significant increase in the latency to initiate trials ( $f$. Baseline data (BL ie, mean of the five sessions immediately preceding each drug treatment session) when the stopsignal presentation were concurrent with the start of the go response $(0 \%)$ are shown for illustrative purposes and were not included in the statistical analysis. **** and * denote $P<0.001$ and $P<0.05$, respectively, for significant pairwise differences between drug treatments.

animal can display inattention with relatively high levels of response control. However, the precise neurobiological basis of the dissociable effects of STS manipulations on attention and response control remains to be established. In the rat five-choice serial reaction time task, lesions of the nucleus basalis magnocellularis (Risbrough et al, 2002) and the pedunculopontine tegmental nucleus (PPTg) (Inglis et al, 2001) have been reported to result in impaired attention and reduced impulsivity, though to our knowledge the effects of equivalent lesions on stop-signal performance have not yet been reported. In rodents, the basal forebrain cholinergic complex projects directly to the cortex, thalamus, and hippocampus, whereas the pontine cholinergic system projects to the thalamus and basal forebrain, and to a lesser extent to the cortex (McKinney and Jacksonville 2005). Given that STS is highly expressed in the mammalian thalamus and cortex (Compagnone et al, 1997; Perumal and Robins 1973; Stergiakouli et al, 2011) ie, key neural substrates in attention and impulsivity (Bonelli and Cummings, 2007; Robbins, 2007), and that both acute DHEAS administration (Rhodes et al, 1996, 1997) and STS inhibition have been shown to influence acetylcholine release, we speculate that STS deficiency may give rise to the observed dissociable pattern of behavioral effects by affecting the integrity or function of cholinergic projections from the basal forebrain and/or the PPTg to the thalamus and cortex.

In addition, or alternatively, STS deficiency could affect attention and impulsivity through effects on monoaminergic function, including serotonergic function (Trent et al, 2012a, 2013), which is increasingly being recognized as an important modulator of inhibitory control (Dalley and Roiser, 2012); interactions between the serotonergic and cholinergic systems, notably within the basal forebrain, are known to be important in mediating cognition (Steckler and Sahgal 1995). STS is expressed in the developing and adult striatum (Compagnone et al, 1997; Perumal and Robins, 1973; Stergiakouli et al, 2011) and, behaviorally, the effects of manipulating the STS axis on response control appeared to be similar in magnitude and pattern to the effects of methylphenidate and atomoxetine; hence, it is also possible that steroid deficiency exerts its inhibitory effects in the stop-signal task in a manner analogous to these drugs ie, through influencing synaptic levels of dopamine and/or noradrenaline in the fronto-striatal regions (Bari and Robbins, 2013). To date, there is no published evidence that STS deficiency influences synaptic levels of these monoamines, although in $39, \mathrm{X}^{\mathrm{Y}^{*}} \mathrm{O}$ mice there is a trend towards increased striatal tissue dopamine concentrations (Trent et al, 2012a). In addition, there is increasing evidence 
for dissociations within, and between, processes of attention and response control mediated by differential effects of monoaminergic transmission occurring in distinct regions of the fronto-striatal circuitry (eg, Economidou et al, 2012; Pezze et al, 2007; Robinson et al, 2008b; Winstanley et al, 2003). However, predictions as to how STS may impact on attention and response control differentially via such brain area-specific monoaminergic mechanisms are hindered by a current lack of knowledge about the functions of the enzyme in discrete components of the fronto-striatal circuitry.

The STS axis, including the direct substrates and products of the enzyme and their downstream effectors, could also in theory be influencing inhibitory and attentional processes in brain via multiple other routes, including via effects on gamma-aminobutyric acid receptor A function, $N$-methyl-Daspartate receptor function or sigma 1 receptor function (Davies, 2012; Reed et al, 2005). Future work examining the behavioral effects of agonists and antagonists at these receptors in $39, \mathrm{X}^{\mathrm{Y}^{*}} \mathrm{O}$ and COUMATE-treated mice will allow us to determine which, if any, of these pathways are mediating the observed STS-dependent effects.

ADHD is a condition underpinned by complex genetic etiology presenting with mixtures of hyperactivity, inattention, and impulsive responding. Subgroups of individuals with the disorder may be differentiated based upon their cognitive profile (Sonuga-Barke et al, 2008). Importantly in the context of the present findings, no clear mediating role of aberrant inhibition in ADHD has yet been demonstrated; indeed, defective information processing, associated with a more cautious response style in some cognitive tasks, has recently been proposed as the key specific mechanism underlying ADHD pathophysiology (Salum et al, 2014). Clearly any one animal model, including those described here, cannot recapitulate the whole of the ADHD spectrum of symptoms, at least in any simple way. However, consistent with our animal data, cytogenetic deletions that include the STS gene (or inactivating point mutations within the gene) are associated with increased risk of developing inattentive subtype ADHD (Kent et al, 2008) whereas polymorphisms within STS are specifically associated with inattentive symptoms rather than impulsive or hyperactive symptoms (Brookes et al, 2008; Stergiakouli et al, 2011). Whether STS-deficient individuals exhibit enhanced response inhibition as we might predict from our present data, and whether these subjects exhibit neural abnormalities in the pathways mentioned above remains to be investigated. Similarly, the extent to which response control is altered in individuals treated with STS inhibitors as a therapy for hormone-dependent cancers (Purohit and Foster, 2012), or in healthy and clinical populations administered DHEA(S) (eg, Grimley Evans et al, 2006; Maninger et al, 2009; Ritsner, 2011) has not yet been explicitly assessed. Overall, our findings suggest that STS deficiency, or pathologies giving rise to analogous neural abnormalities, may contribute towards the pathophysiology of ADHD cases characterized by inattention with normal (or enhanced) response inhibition.

In conclusion, converging genetic and pharmacological evidence indicates that the STS axis is involved in inhibitory processes and can be manipulated to give rise to highly specific and substantial improvements in response control.
Additional work specifying neurobiological changes elicited by STS deficiency (eg, Trent et al, 2014) is needed to fully understand the mechanism(s) of action by which such manipulations impact upon dissociable components of attention and response control. We suggest the steroid sulfate axis, or more likely its downstream effectors acting on inhibitory processes, might represent viable therapeutic targets for the treatment of ADHD and other disorders where failures in behavioral inhibition are prominent.

\section{FUNDING AND DISCLOSURE}

The authors declare no conflict of interest.

\section{ACKNOWLEDGEMENTS}

The work was supported by a Cardiff University LINK Award to LSW, by a Medical Research Council United Kingdom (MRC, UK) New Investigator Research Grant to WD (G0900636), by a Research Councils UK Fellowship to WD, and by MRC funding (U117532009) to OAO. We thank Dr Jonathan Fry for the gift of COUMATE and Clive Mann, Pat Mason and other members of JBIOS, Cardiff University for expert animal husbandry.

\section{Disclaimer}

The funding bodies had no role in the design of the study, in the collection and analysis of data or in the decision to publish.

\section{REFERENCES}

Aron AR, Dowson JH, Sahakian BJ, Robbins TW (2003). Methylphenidate improves response inhibition in adults with attention-deficit/hyperactivity disorder. Biol Psychiatry 54: 1465-1468.

Bari A, Robbins TW (2013). Noradrenergic versus dopaminergic modulation of impulsivity, attention and monitoring behaviour in rats performing the stop-signal task: possible relevance to ADHD. Psychopharmacology 230: 89-111.

Blondeau C, Dellu-Hagedorn F (2007). Dimensional analysis of ADHD subtypes in rats. Biol Psychiatry 61: 1340-1350.

Bonelli RM, Cummings JL (2007). Frontal-subcortical circuitry and behavior. Dialogues Clin Neurosci 9: 141-151.

Brewer JA, Potenza MN (2008). The neurobiology and genetics of impulse control disorders: relationships to drug addictions. Biochem Pharmacol 75: 63-75.

Brookes KJ, Hawi Z, Kirley A, Barry E, Gill M, Kent L (2008). Association of the steroid sulfatase (STS) gene with attention deficit hyperactivity disorder. Am J Med Genet B Neuropsychiatr Genet 147B: 1531-1535.

Brookes KJ, Hawi Z, Park J, Scott S, Gill M, Kent L (2010). Polymorphisms of the steroid sulfatase (STS) gene are associated with attention deficit hyperactivity disorder and influence brain tissue mRNA expression. Am J Med Genet B Neuropsychiatr Genet 153B: 1417-1424.

Carter JD, Farrow M, Silberstein RB, Stough C, Tucker A, Pipingas A (2003). Assessing inhibitory control: a revised approach to the stop signal task. J Atten Disord 6: 153-161.

Chamberlain SR, Muller U, Blackwell AD, Clark L, Robbins TW, Sahakian BJ (2006). Neurochemical modulation of response inhibition and probabilistic learning in humans. Science 311: 861-863. 
Compagnone NA, Salido E, Shapiro LJ, Mellon SH (1997). Expression of steroid sulfatase during embryogenesis. Endocrinology 138: 4768-4773.

Crosbie J, Perusse D, Barr CL, Schachar RJ (2008). Validating psychiatric endophenotypes: inhibitory control and attention deficit hyperactivity disorder. Neurosci Biobehav Rev 32: 40-55.

Dalley JW, Roiser JP (2012). Dopamine, serotonin and impulsivity. Neuroscience 215: 42-58.

Davies W (2012). Does steroid sulfatase deficiency influence postpartum psychosis risk? Trends Mol Med 18: 256-262.

Davies W, Humby T, Isles AR, Burgoyne PS, Wilkinson LS (2007). $\mathrm{X}$-monosomy effects on visuospatial attention in mice: a candidate gene and implications for Turner syndrome and attention deficit hyperactivity disorder. Biol Psychiatry 61: 1351-1360.

Davies W, Humby T, Kong W, Otter T, Burgoyne PS, Wilkinson LS (2009). Converging pharmacological and genetic evidence indicates a role for steroid sulfatase in attention. Biol Psychiatry 66: $360-367$

DeVito EE, Blackwell AD, Clark L, Kent L, Dezsery AM, Turner DC et al (2009). Methylphenidate improves response inhibition but not reflection-impulsivity in children with attention deficit hyperactivity disorder (ADHD). Psychopharmacology (Berl) 202: 531-539.

Eagle DM, Robbins TW (2003). Lesions of the medial prefrontal cortex or nucleus accumbens core do not impair inhibitory control in rats performing a stop-signal reaction time task. Behav Brain Res 146: 131-144.

Eagle DM, Tufft MR, Goodchild HL, Robbins TW (2007). Differential effects of modafinil and methylphenidate on stopsignal reaction time task performance in the rat, and interactions with the dopamine receptor antagonist cis-flupenthixol. Psychopharmacology (Berl) 192: 193-206.

Economidou D, Theobald DE, Robbins TW, Everitt BJ, Dalley JW (2012). Norepinephrine and dopamine modulate impulsivity on the five-choice serial reaction time task through opponent actions in the shell and core sub-regions of the nucleus accumbens. Neuropsychopharmacology 37: 2057-2066.

Grimley Evans J, Malouf R, Huppert F, van Niekerk JK (2006). Dehydroepiandrosterone (DHEA) supplementation for cognitive function in healthy elderly people. Cochrane Database Syst Rev 4: CD006221.

Hart H, Radua J, Nakao T, Mataix-Cols D, Rubia K (2013). Metaanalysis of functional magnetic resonance imaging studies of inhibition and attention in attention-deficit/hyperactivity disorder: exploring task-specific, stimulant medication, and age effects. JAMA Psychiatry 70: 185-198.

Holden C (2005). Sex and the suffering brain. Science 308: 1574.

Humby T, Eddy JB, Good MA, Reichelt AC, Wilkinson LS (2013). A novel translational assay of response inhibition and impulsivity: effects of prefrontal cortex lesions, drugs used in ADHD, and serotonin $2 \mathrm{C}$ receptor antagonism. Neuropsychopharmacology 38: $2150-2159$.

Humby T, Wilkinson LS (2011). Assaying dissociable elements of behavioural inhibition and impulsivity: translational utility of animal models. Curr Opin Pharmacol 11: 534-539.

Inglis WL, Olmstead MC, Robbins TW (2001). Selective deficits in attentional performance on the 5-choice serial reaction time task following pedunculopontine tegmental nucleus lesions. Behav Brain Res 123: 117-131.

Kent L, Emerton J, Bhadravathi V, Weisblatt E, Pasco G, Willatt LR et al (2008). X-linked ichthyosis (steroid sulfatase deficiency) is associated with increased risk of attention deficit hyperactivity disorder, autism and social communication deficits. J Med Genet 45: $519-524$

Logan GD (1994). On the ability to inhibit thought and action: a users' guide to the stop signal paradigm. In: DC TH (ed).
Inhibitory Processes in Attention, Memory and Language. Academic Press: San Diego, pp 189-239.

Logan GD, Cowan WB, Davis KA (1984). On the ability to inhibit simple and choice reaction time responses: a model and a method. J Exp Psychol Hum Percept Perform 10: 276-291.

Maninger N, Wolkowitz OM, Reus VI, Epel ES, Mellon SH (2009). Neurobiological and neuropsychiatric effects of dehydroepiandrosterone (DHEA) and DHEA sulfate (DHEAS). Front Neuroendocrinol 30: 65-91.

McKinney M, Jacksonville MC (2005). Brain cholinergic vulnerability: Relevance to behavior and disease. Biochem Pharmacol 70: $1115-1124$.

Nicolas LB, Fry JP (2007). The steroid sulfatase inhibitor COUMATE attenuates rather than enhances access of dehydroepiandrosterone sulfate to the brain in the mouse. Brain Res 1174: 92-96.

Nicolas LB, Pinoteau W, Papot S, Routier S, Guillaumet G, Mortaud S (2001). Aggressive behavior induced by the steroid sulfatase inhibitor COUMATE and by DHEAS in CBA/H mice. Brain Res 922: 216-222.

Perumal AS, Robins E (1973). Regional and subcellular distribution of aryl-and steroid sulfatases in brain. Brain Res 59: 349358.

Pezze MA, Dalley JW, Robbins TW (2007). Differential roles of dopamine D1 and D2 receptors in the nucleus accumbens in attentional performance on the five-choice serial reaction time task. Neuropsychopharmacology 32: 273-283.

Purohit A, Foster PA (2012). Steroid sulfatase inhibitors for estrogen- and androgen-dependent cancers. J Endocrinol 212: 99-110.

Reed MJ, Purohit A, Woo LW, Newman SP, Potter BV (2005). Steroid sulfatase: molecular biology, regulation, and inhibition. Endocr Rev 26: 171-202.

Rhodes ME, Li PK, Burke AM, Johnson DA (1997). Enhanced plasma DHEAS, brain acetylcholine and memory mediated by steroid sulfatase inhibition. Brain Res 773: 28-32.

Rhodes ME, Li PK, Flood JF, Johnson DA (1996). Enhancement of hippocampal acetylcholine release by the neurosteroid dehydroepiandrosterone sulfate: an in vivo microdialysis study. Brain Res 733: 284-286.

Risbrough V, Bontempi B, Menzaghi F (2002). Selective immunolesioning of the basal forebrain cholinergic neurons in rats: Effect on attention using the 5-choice serial reaction time task. Psychopharmacology (Berl) 164: 71-81.

Ritsner MS (2011). The clinical and therapeutic potentials of dehydroepiandrosterone and pregnenolone in schizophrenia. Neuroscience 191: 91-100.

Robbins TW (2002). The 5-choice serial reaction time task: behavioural pharmacology and functional neurochemistry. Psychopharmacology 163: 362-380.

Robbins TW (2007). Shifting and stopping: fronto-striatal substrates, neurochemical modulation and clinical implications. Philos Trans R Soc Lond B Biol Sci 362: 917-932.

Robinson ES, Dalley JW, Theobald DE, Glennon JC, Pezze MA, Murphy ER et al (2008b). Opposing roles for 5-HT2A and 5HT2C receptors in the nucleus accumbens on inhibitory response control in the 5-choice serial reaction time task. Neuropsychopharmacology 33: 2398-2406.

Robinson ES, Eagle DM, Mar AC, Bari A, Banerjee G, Jiang X et al (2008a). Similar effects of the selective noradrenaline reuptake inhibitor atomoxetine on three distinct forms of impulsivity in the rat. Neuropsychopharmacology 33: 1028-1037.

Salum GA, Sergeant J, Sonuga-Barke E, Vandekerckhove J, Gadelha A, Pan PM et al (2014). Specificity of basic information processing and inhibitory control in attention deficit hyperactivity disorder. Psychol Med 44: 617-631, 1-15.

Solanto MV, Abikoff H, Sonuga-Barke E, Schachar R, Logan GD, Wigal $\mathrm{T}$ et al (2001). The ecological validity of delay aversion 
and response inhibition as measures of impulsivity in AD/HD: a supplement to the NIMH multimodal treatment study of AD/HD. J Abnorm Child Psychol 29: 215-228.

Sonuga-Barke EJ, Sergeant JA, Nigg J, Willcutt E (2008). Executive dysfunction and delay aversion in attention deficit hyperactivity disorder: nosologic and diagnostic implications. Child Adolesc Psychiatr Clin N Am 17: 367-384.

Steckler T, Sahgal A (1995). The role of serotonergic-cholinergic interactions in the mediation of cognitive behaviour. Behav Brain Res 67: 165-199.

Stergiakouli E, Langley K, Williams H, Walters J, Williams NM, Suren $S$ et al (2011). Steroid sulfatase is a potential modifier of cognition in attention deficit hyperactivity disorder. Genes Brain Behav 10: 334-344.

Tannock R, Schachar RJ, Carr RP, Chajczyk D, Logan GD (1989). Effects of methylphenidate on inhibitory control in hyperactive children. J Abnorm Child Psychol 17: 473-491.

Thapar A, O'Donovan M, Owen MJ (2005). The genetics of attention deficit hyperactivity disorder. Hum Mol Genet 14(Suppl 2): R275-R282.
Trent S, Cassano T, Bedse G, Ojarikre OA, Humby T, Davies W (2012a). Altered serotonergic function may partially account for behavioral endophenotypes in steroid sulfatase-deficient mice. Neuropsychopharmacology 37: 1267-1274.

Trent S, Dean R, Veit B, Cassano T, Bedse G, Ojarikre OA et al (2013). Biological mechanisms associated with increased perseveration and hyperactivity in a genetic mouse model of neurodevelopmental disorder. Psychoneuroendocrinology 38: 1370-1380.

Trent S, Dennehy A, Richardson H, Ojarikre OA, Burgoyne PS, Humby $\mathrm{T}$ et al (2012b). Steroid sulfatase-deficient mice exhibit endophenotypes relevant to attention deficit hyperactivity disorder. Psychoneuroendocrinology 37: 221-229.

Trent S, Fry JP, Ojarikre OA, Davies W (2014). Altered brain gene expression but not steroid biochemistry in a genetic mouse model of neurodevelopmental disorder. Mol Autism 5: 21.

Winstanley CA, Chudasama Y, Dalley JW, Theobald DE, Glennon JC, Robbins TW (2003). Intra-prefrontal 8-OH-DPAT and M100907 improve visuospatial attention and decrease impulsivity on the five-choice serial reaction time task in rats. Psychopharmacology 167: 304-314.

Supplementary Information accompanies the paper on the Neuropsychopharmacology website (http://www.nature.com/npp) 